Research Article

\title{
Structural Reanalysis Based on FRFs Using Sherman-Morrison-Woodbury Formula
}

\author{
Jun Ren (iD) and Qianghao Zhang \\ School of Mechanical Engineering, Hubei University of Technology, Wuhan, Hubei 430068, China \\ Correspondence should be addressed to Jun Ren; renjun@mail.hbut.edu.cn
}

Received 20 December 2019; Revised 28 May 2020; Accepted 25 June 2020; Published 31 July 2020

Academic Editor: Hamid Toopchi-Nezhad

Copyright (C) 2020 Jun Ren and Qianghao Zhang. This is an open access article distributed under the Creative Commons Attribution License, which permits unrestricted use, distribution, and reproduction in any medium, provided the original work is properly cited.

\begin{abstract}
Structural dynamic modification is a popular approach to obtain desire frequencies and dynamic characteristics. It has been observed that reanalyzing the modified structure usually involves complicated calculations when modifications are concerned with numerous degrees of freedom (DOFs), especially adding substructures to these DOFs. This paper proposed a method to reanalyze the frequency response functions (FRFs) of structures with multiple co-ordinates modifications. Two different cases are taken into consideration in the modifications, including adding (or decreasing) masses, stiffness, and damping, as well as adding spring-mass substructures, which makes the method more practical. This method is developed by employing Sherman-Morrison and Woodbury (SMW) formula based on the FRFs related to the modifications coordinates of the original system. The advantage of this method is that neither a physical model nor a modal model is required; instead, it needs only the FRFs, which can be directly measured by experimental modal testing. Another salient feature of this proposed strategy is that the FRFs of the modified structure can be calculated in only one step. Validation of this proposed method is demonstrated using various numerical examples. It is shown that the method is very effective and can be considered for real applications.
\end{abstract}

\section{Introduction}

Structural dynamic modification has been applied widely in practical engineering. In many engineering cases, structural dynamic modifications are used to obtain better dynamic characteristics of structures. Generally speaking, structural dynamic modification refers to a method to get certain structural dynamic characteristics by changing the local physical parameters (masses, stiffness, and damping), such as the need to avoid resonance or assignment of certain frequencies on desired locations. Structural dynamic modification is an economical and effective means of improving the dynamic characteristics of mechanical structures. This approach is widely used in aerospace, marine, automotive, civil engineering, bridge, and machinery industries.

The problems involved in structural dynamic modification can be divided into two categories: "forward problem" and "inverse problem." The former aims to predict exact change to the structure's dynamic properties when known modifications are made at a given location $[1,2]$. The latter mainly studies how to modify an existing structure in order to achieve the expected dynamic characteristics (such as natural frequency and mode shape) [3-6]. The forward structural dynamic modification is also called structural reanalysis in early studies. In structural reanalysis, the dynamic effects of a modification on a structure are treated as an analysis problem involving the known dynamic properties of the original structure rather than a complete reanalysis of the modified structure [7]. This approach could avoid the blindness of the design, which reduces the design cost and has practical engineering application value.

Many works and literatures had conducted a comprehensive analysis of reanalysis methods. Reanalysis methods can be generally divided into two categories: direct methods and approximate methods. The approximate methods can be divided into three categories [8]: global approximation, local approximation, and combined approximation. 
For the development of direct methods, early studies of structural reanalysis were reviewed and summarized by Baldwin and Hutton [7]. Several approaches such as Rayleigh quotient [9], sensitivity analysis [10], and perturbation approach [11] were used to address forward modification problems without a complete reanalysis of the whole structure.

This important issue has also been extensively discussed in recent years, and part of the relevant literature is summarized as follows. This problem was explored in [12] using developed successive matrix inverse method based on symmetry of corresponding stiffness matrix after constraint modification of the boundary. The numerical examples show that this method could quickly give accurate reanalysis results. In the same year, Liu et al. [13] proposed an approach for structural static reanalysis with unchanged number of degrees of freedom. This approach was based on a new preconditioner constructed by updating the Cholesky factorization of the original stiffness matrix, which could achieve fast convergence and accurate results. After two years, Song et al. [14] suggested using a direct reanalysis algorithm based on finding updated triangular factorization in sparse matrix solution to solve this problem. This algorithm is suitable for local modification, and the examples show that the algorithm improves reanalysis efficiency significantly, especially for high-rank structural modification. Later on, a "cheap" algorithm, named independent coefficients method, was put forward to reanalyze structures with local modification, which leads to a low-rank change in the stiffness matrix [15]. Considering that previous work [13] suffers limitation of a structure as added degrees of freedom, a new and efficient reanalysis method [16] had been proposed by the same author. Another advantage of this method is that the Cholesky factorization of the stiffness matrix of the modified structure can be used as the initial information for reanalysis when the structure is further modified. In the work of [17], the issue was suggested using a nonlinear reanalysis method based on structural modification of residual incremental approximations. In contrast to other existing nonlinear reanalysis methods, which were based on the evaluation of changed stiffness matrices, only residual vectors need to be computed and stored. Kim and Eun [18] studied coupling and recoupling reanalysis methods. These methods were performed by using the concept of compatibility conditions at interface nodes between the substructures or between the original structure and the substructures. The most recent theory proposed in [19] offered a new method for free-vibration reanalysis after structural topological modifications with added degrees of freedom. The implementation of this approach involved only $\mathrm{LDL}^{\mathrm{T}}$ factorization of shifted substiffness matrices corresponding to the newly added DOFs, and the proposed method consists of matrix-matrix operations.

As for approximate methods, the combined approximation method is an effective solution method that combines the high efficiency of the local approximation method with the high quality of the global approximation method. The solution process of the method is based on the results of an accurate single-point analysis, and it is also a reanalysis method that has developed very fast in recent years. The original purpose of studying the CA method is to accelerate the optimization design. CA method has been widely used in many fields such as linear or nonlinear static analysis, dynamic analysis, modal analysis, and sensitivity analysis [8].

For the combined approximation method of reanalysis, Kirsch [20] first used forward and reverse substitution calculations to calculate the terms used as the basis vector binomial sequence in the CA method solving process. A new set of uncoupled basis vectors are generated and normalized by using the Gram-Schmidt orthogonalization process. This method can achieve an effective and accurate approximation for very large design changes. By 2006, Kirsch et al. [21] used combined approximation method to overcome the repeated eigenproblem solution of nonlinear dynamic reanalysis and solved its main problem. The method is based on the integration of several concepts and methods, including the basis of matrix factorization, series expansion, and reduction. In order to solve the frequency-constrained structural optimization problem, Zuo et al. [22] proposed an adaptive eigenvalue reanalysis method based on genetic algorithm for structural optimization. The modified impulse analysis method is a combination approximation method from Kirsch, and it has a high level for repeated eigenvalue problems accuracy. Considered to integrate the Kirsch's method into the result optimization process, a new adaptive method [23] that used the $K$ condition number to determine the minimum number of basis vectors was proposed. Besides, on the reanalysis of sensitivity, Zuo et al. [24] proposes a new method for arbitrarily changing static displacement sensitive design variables. This method uses Taylor series expansion to approximate the current displacement of the modified sensitivity equation and then solves the direct sensitivity equation by a combined approximation method. One year later, the same author [25] conducted a sensitivity analysis of eigenvalues and eigenvectors using a combination approximation method, and the eigenvectors were solved by the Nelson method. This method can greatly improve the efficiency of sensitivity analysis and can accelerate the gradient-based structural optimization constraints with frequency and mode shape.

All these researches mentioned above are primarily based on the physical model, which requires the knowledge of mass, stiffness, and damping matrices. In practical engineering, however, these parameters matrices of vibration system structures are not easy to obtain. This is because the structure to be modified is usually a complex structure with multiple DOFs. Furthermore, in the process of reanalysis calculation, it is usually not very difficult to solve the singleelement change problem. When it comes to multiple-element change structures, however, the calculation becomes more complicated. Therefore, these problems limit the application of the above methods to some extent.

This paper proposed a method for reanalyzing FRFs of the modified structure. This method is developed by employing SMW formula $[26,27]$ based on the FRFs related to modifications coordinates of the original system. The advantage of this FRFs-based method is that the FRFs can be directly measured by model testing, without knowledge of 
the system matrices $M, C, K$, which are usually unavailable in practical engineering. Another salient feature of this proposed strategy is that the FRFs of a structure with multipleelements changing can be calculated in only one step, which improves the efficiency of reanalysis.

\section{Theoretical Development}

The equation of motion of a free-vibration damping multidegree-of-freedom system can be expressed as

$$
M \ddot{x}+C \dot{x}+K=0 .
$$

The dynamic stiffness matrix of original structure can be given by

$$
Z=K-M \omega^{2}+j \omega C
$$

where $Z$ is the dynamic stiffness matrix of original structure; $\omega$ represents the frequency variable and $j=\sqrt{-1}$.

2.1. Adding Masses, Stiffness, and Damping to the Original Model Structure. It is assumed that the local modification of the structure involves $n$ coordinate points, labeled $1,2, \ldots, n$. The additional masses, stiffnesses, and damping at these points are denoted as $\Delta m_{1}, \Delta m_{2}, \ldots, \Delta m_{n} ; \Delta k_{1}, \Delta k_{2}, \ldots, \Delta k_{n}$; $\Delta c_{1}, \Delta c_{2}, \ldots, \Delta c_{n}$, respectively. The values of these additional stiffnesses, additional masses, and additional damping can be positive or negative. If the value is positive, then additional parameters are added to the original structure, while a negative value means that additional parameters are reduced from the original structure.

The above additional masses, stiffnesses, and damping can be expressed as the diagonal matrix of (3), (4), and (5), respectively.

$$
\begin{aligned}
& \Delta M=\left[\begin{array}{lll}
\Delta m_{1} & & \\
& \ddots & \\
& & \Delta m_{n}
\end{array}\right], \\
& \Delta K=\left[\begin{array}{lll}
\Delta k_{1} & & \\
& \ddots & \\
& & \Delta k_{n}
\end{array}\right], \\
& \Delta C=\left[\begin{array}{lll}
\Delta c_{1} & & \\
& \ddots & \\
& & \Delta c_{n}
\end{array}\right] .
\end{aligned}
$$

After adding additional masses $\Delta M$, additional stiffnesses $\Delta K$, and additional damping $\Delta C$,

$$
Z^{*}=K+\Delta K-(M+\Delta M) \omega^{2}+j \omega(C+\Delta C)=Z+\Delta Z,
$$

where $Z^{*}$ is the structure dynamic stiffness matrix after adding additional parameters to original structure.

$$
\Delta Z=\Delta K-\Delta M \omega^{2}+j \omega \Delta C
$$

To make $\Delta Z$ more intuitive, it is expressed by the following formula:

$$
\Delta Z=\sum_{k=1}^{n} U_{k} V_{k}^{T},
$$

where $U_{k}$ is a column vector of $n \times 1$ in which element of the $k_{\mathrm{th}}$ row is 1 , and the other elements are zero. $V_{\mathrm{k}}$ represents a column vector of $n \times 1$ in which element of the $k_{\text {th }}$ row is and the other elements are zero. Then the column vectors $U$ and $V$ can be given by

$$
U_{1}=\left[\begin{array}{c}
1 \\
0 \\
\vdots \\
0
\end{array}\right], \ldots, U_{n}=\left[\begin{array}{c}
0 \\
0 \\
\vdots \\
1
\end{array}\right] V_{1}=\left[\begin{array}{c}
\Delta k_{1}-\Delta m_{1} \omega^{2}+j \omega \Delta c_{1} \\
0 \\
\vdots \\
0
\end{array}\right], \ldots, V_{N}=\left[\begin{array}{c}
0 \\
0 \\
\vdots \\
\Delta k_{n}-\Delta m_{n} \omega^{2}+j \omega \Delta c_{n}
\end{array}\right]
$$

According to SMW formula [28], the dynamic stiffness $Z^{*-1}$ of modified structure with multielement change is simply written as

$$
\begin{aligned}
Z^{*-1}= & (Z+\Delta Z)^{-1}=Z^{-1}-Z^{-1}\left[\begin{array}{lll}
U_{1} & \cdots & U_{N}
\end{array}\right] W^{-1} \\
& \cdot\left[\begin{array}{lll}
V_{1}^{T} & \cdots & V_{N}^{T}
\end{array}\right]^{T} Z^{-1}
\end{aligned}
$$

$$
W=\left[\begin{array}{cccc}
1+V_{1}^{T} H U_{1} & V_{1}^{T} H U_{2} & \cdots & V_{1}^{T} H U_{n} \\
V_{2}^{T} H U_{1} & \ddots & & \vdots \\
\vdots & & \ddots & \\
V_{n}^{T} H U_{1} & \cdots & & 1+V_{n}^{T} H U_{n}
\end{array}\right]
$$

Since the dynamic stiffness matrix of structure and the FRFs matrix are inverse matrices of each other, then

$$
Z^{*-1}=H^{*}=H-H\left[\begin{array}{llll}
U_{1} & \cdots & U_{N}
\end{array}\right] W^{-1}\left[\begin{array}{lll}
V_{1}^{T} & \cdots & V_{N}^{T}
\end{array}\right]^{T} H,
$$

where 
where $H$ is FRFs matrix of original structure; $H^{*}$ is FRFs matrix of the structure after adding additional masses, stiffnesses, and damping.

It is obvious that the FRFs matrix $H^{*}$ can be calculated according to (12) when the FRFs $H$ of original structure and added masses matrix $\Delta M$, stiffnesses matrix $\Delta K$, and damping matrix $\Delta C$ are known.

It should be noted that the FRFs matrices $H$ and $H^{*}$ mentioned above are displacement FRFs matrices. In the practical engineering cases, acceleration sensors are usually used to measure the response, so accelerance $\mathrm{Ha}$ is directly obtained. The relationship between $H$ and $H a$ can be described as

$$
H a=-\omega^{2} H
$$

Therefore, once accelerance matrix $\mathrm{Ha}$ of the original structure is obtained in practical application, the receptances $H$ can be calculated according to (13), then substituting it into (10) and (11) to calculate receptances $H^{*}$ of the modified structure, where $H^{*}$ could be expressed as

$$
H^{*}=H-H\left[\begin{array}{lll}
U_{1} & \cdots & U_{N}
\end{array}\right] W^{-1}\left[\begin{array}{lll}
V_{1}^{T} & \cdots & V_{1}^{T}
\end{array}\right]^{T} H .
$$

Finally, the obtained $H^{*}$ is substituted into (13) to calculate accelerances $\mathrm{Ha}^{*}$ of the modified structure.

2.2. Adding Spring-Mass Substructures to the Original Structure. In many engineering cases, the vibration system structures are designed to be immutable. When original structure does not satisfy the dynamic characteristics, adding spring-mass substructures to the original structural system is not a bad idea. The added spring-mass substructure is shown in Figure 1.
For the reason of adding a spring-mass substructure, the DOFs of original system has been changed, and one DOF is added to original system. The above calculated method is not suitable for this kind of condition anymore, and thus transforming the DOFs of modified structure to ones of original structure is needed [29].

The equation of motion of a linear undamped multidegree-of-freedom system can be expressed as

$$
M \ddot{x}+K x=f .
$$

Assuming that the mass and stiffness modification of original system are $\Delta M, \Delta K$, then (15) can be written as

$$
(M+\Delta M) \ddot{x}+(K+\Delta K) x=f .
$$

Assuming harmonic response $x=u e^{i \omega t}$ and substituting it into (15), dynamic stiffness matrix $Z$ and FRFs $H$ of the system can be written as follows:

$$
\begin{aligned}
& Z(\omega)=-\omega^{2} M+K, \\
& H(\omega)=\left(-\omega^{2} M+K\right)^{-1} .
\end{aligned}
$$

Then (16) yields

$$
H^{-1} u=\left(\omega^{2} \Delta M-\Delta K\right) u+f .
$$

It is assumed that a spring-mass substructure is added at the $i_{\text {th }}$ freedom of original structure. The mass and stiffness are $\Delta m, \Delta k$ and the relative amplitude of vibration is $\Delta u$. Since one substructure is added to the original system structure, an extra freedom is added. The DOFs of original structure are changed from $n$ to $n+1$, and the matrices in (18) are enlarged by one row and column. Then, the equation of motion of the modified system is described by

$$
\left(\begin{array}{cc}
H_{n \times n}^{-1} & 0 \\
0 & 0
\end{array}\right)\left(\begin{array}{c}
u_{1} \\
u_{2} \\
\vdots \\
u_{i} \\
\vdots \\
u_{n} \\
\Delta u
\end{array}\right)=\left(\begin{array}{cccccccc}
0 & 0 & \cdots & 0 & \cdots & 0 & 0 \\
0 & 0 & \cdots & 0 & \cdots & 0 & 0 \\
\vdots & \vdots & \ddots & \vdots & & \vdots & \vdots \\
0 & 0 & \cdots & -\Delta k & \cdots & 0 & \Delta k \\
\vdots & \vdots & & \vdots & \ddots & \vdots & \vdots \\
0 & 0 & \cdots & 0 & \cdots & 0 & 0 \\
0 & 0 & \cdots & \Delta k & \cdots & 0 & -\Delta k+\omega^{2} \Delta m
\end{array}\right)\left(\begin{array}{c}
u_{1} \\
u_{2} \\
\vdots \\
u_{i} \\
\vdots \\
u_{n} \\
\Delta u
\end{array}\right)+\left(\begin{array}{c}
f_{1} \\
f_{2} \\
\vdots \\
f_{i} \\
\vdots \\
f_{n} \\
0
\end{array}\right) .
$$

The last row of above equation is

$$
\Delta k u_{i}+\left(-\Delta k+\omega^{2} \Delta m\right) \Delta u=0
$$

Substitute $\Delta u$ with $u_{i}$

$$
\Delta u=\left(\frac{\Delta k}{\Delta k-\omega^{2} \Delta m}\right) u_{i} .
$$

Substituting (21) into (19), the right matrix of (19) can be written as follows: 




FIgure 1: Adding a spring-mass substructure on the $i_{\text {th }}$ coordinate of the $n$ DOF system.

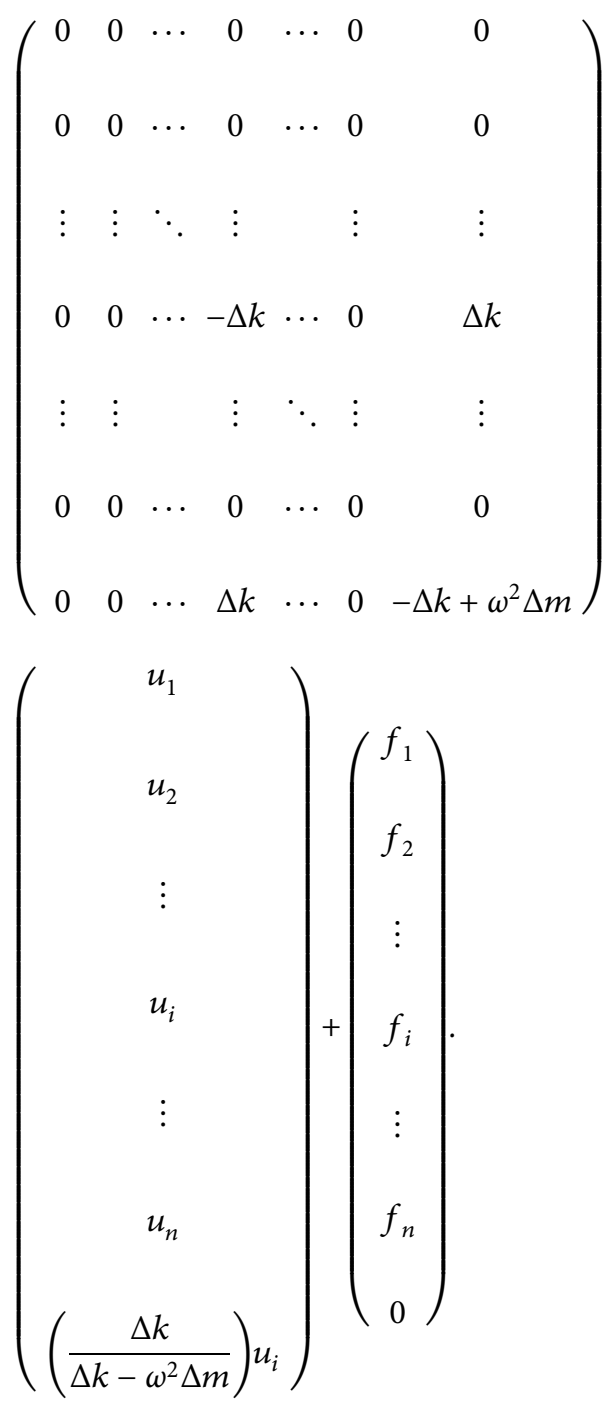

Considering the $i_{\text {th }}$ and $(n+1)_{\text {th }}$ row of $(22)$, one can obtain

$$
\begin{array}{r}
-\Delta k u_{i}+\left(\frac{(\Delta k)^{2}}{\Delta k-\omega^{2} \Delta m}\right) u_{i}+f_{i}=\left(\frac{\omega^{2} \Delta m \Delta k}{\Delta k-\omega^{2} \Delta m}\right) u_{i}+f_{i} \\
\Delta k u_{i}+\left(\frac{\omega^{2} \Delta m-\Delta k}{\Delta k-\omega^{2} \Delta m}\right) \Delta k u_{i}=0 .
\end{array}
$$

Then (19) can be written as follows:

$$
\begin{aligned}
H_{n \times n}^{-1}\left(\begin{array}{c}
u_{1} \\
u_{2} \\
\vdots \\
u_{i} \\
\vdots \\
u_{n}
\end{array}\right)=\left(\begin{array}{cccccc}
0 & 0 & \cdots & 0 & \cdots & 0 \\
0 & 0 & \cdots & 0 & \cdots & 0 \\
\vdots & \vdots & \ddots & \vdots & & \vdots \\
0 & 0 & \cdots & \frac{\omega^{2} \Delta m \Delta k}{\Delta k-\omega^{2} \Delta m} & \cdots & 0 \\
\vdots & \vdots & & \vdots & \ddots & \vdots \\
0 & 0 & \cdots & 0 & \cdots & 0
\end{array}\right)\left(\begin{array}{c}
u_{1} \\
u_{2} \\
\vdots \\
u_{i} \\
\vdots \\
u_{n}
\end{array}\right) \\
+\left(\begin{array}{c}
f_{1} \\
f_{2} \\
\vdots \\
f_{i} \\
\vdots \\
f_{n}
\end{array}\right) .
\end{aligned}
$$

It is easy to observe from (24) that when adding one spring-mass substructure at the $i_{\text {th }}$ freedom of the original structure, element in the $i_{\text {th }}$ row represented by $V_{k}$ in the (8) is $-\left(\omega^{2} \Delta m_{k} \Delta k_{k} / \Delta k_{k}-\omega^{2} \Delta m_{k}\right)$ in this situation, and the other elements of $V_{\mathrm{k}}$ are zero; namely,

$$
V_{1}=\left(\begin{array}{c}
-\frac{\omega^{2} \Delta m_{1} \Delta k_{1}}{\Delta k_{1}-\omega^{2} \Delta m_{1}} \\
0 \\
\vdots \\
0
\end{array}\right), \cdots, V_{n}=\left(\begin{array}{c}
0 \\
0 \\
\vdots \\
-\frac{\omega^{2} \Delta m_{n} \Delta k_{n}}{\Delta k_{n}-\omega^{2} \Delta m_{n}}
\end{array}\right) \text {. }
$$

Without changing original structure of the system and adding one substructure to a certain coordinate of the system, after added one substructure, the DOFs of original system structure are changed from $n$ to $n+1$. According to a series of numerical operations, the DOFs of the system structure after adding one substructure are transformed from $n+1$ to $n$, which could meet the calculation approach proposed in this paper.

\section{Verification of the Method}

In this section, three simulated examples are analyzed by the proposed method. 
3.1. Numerical Experiment Setup. To verify the accuracy of the above method, a cantilever beam modal test model is constructed as shown in Figure 2. The physical parameters are shown in Table 1 . The cantilever beam is discretely divided into 6 equal parts along the length direction, and 6 measuring points are evenly distributed.

As shown in Figure 3. Ha-ori-lp are the accelerances curves of original structure, where $H a_{1 \mathrm{p}}$ are calculated accelerances relating points $l$ and $p$.

Two kinds of examples are given to prove this approach proposed in this paper: one is to directly increase or decrease masses, stiffnesses, or damping on original structure. The other is to add a spring-mass substructure to a certain coordinate or multiple coordinates to the original structure. What should be pointed out is that the substructures are added in the vertical direction of the cantilever. For comparison purpose, exact accelerances $\mathrm{Ha}$ corresponding with modified structure are also numerically calculated.

3.2. Adding Mass, Stiffness, and Damping to the Original Model Structure. As shown in Figure 4, the additional stiffness and damping are added at coordinates 2 and 4 , stiffnesses $\Delta k_{2}$ and $\Delta k_{4}$ are $6000 \mathrm{~N} / \mathrm{m}$ and $8000 \mathrm{~N} / \mathrm{m}$, respectively, and damping $\Delta c_{2}$ and $\Delta c_{4}$ are $30 \mathrm{Ns} / \mathrm{m}$ and $20 \mathrm{Ns} /$ $\mathrm{m}$, respectively. Additional masses are added at coordinates 2,4 , and 6, respectively, and the additional masses $\Delta m_{2}, \Delta m_{4}$ and $\Delta m_{6}$ are $0.38 \mathrm{Kg}, 0.42 \mathrm{Kg}$, and $0.4 \mathrm{Kg}$, respectively. Response points are chosen at coordinates 2, 4, and 6 . Hammer impact is moving sequentially from points 2, 4 to 6 for "measuring" accelerances $\mathrm{Ha}=\left[\begin{array}{lll}H a_{22} & H a_{24} & H a_{26} \\ H a_{42} & H a_{44} & H a_{46} \\ H a_{62} & H a_{64} & H a_{66}\end{array}\right]$.

The goal of this example is to calculate the accelerances $H a^{*}=\left[\begin{array}{lll}H a_{22}^{*} & H a_{24}^{*} & H a_{26}^{*} \\ H a_{42}^{*} & H a_{44}^{*} & H a_{46}^{*} \\ H a_{62}^{*} & H a_{64}^{*} & H a_{66}^{*}\end{array}\right]$.

According to (3), (4), and (5), the additional mass matrix, stiffness matrix, and damping matrix in this example are

$$
\begin{aligned}
& \Delta M=\left[\begin{array}{lll}
m_{2} & & \\
& m_{4} & \\
& & m_{6}
\end{array}\right]=\left[\begin{array}{lll}
0.38 & & \\
& 0.42 & \\
& & 0.4
\end{array}\right], \\
& \Delta K=\left[\begin{array}{lll}
k_{2} & & \\
& k_{4} & \\
& & k_{6}
\end{array}\right]=\left[\begin{array}{lll}
6000 & & \\
& 8000 & \\
& & 0
\end{array}\right], \\
& \Delta C=\left[\begin{array}{lll}
c_{2} & & \\
& c_{4} & \\
& & c_{6}
\end{array}\right]=\left[\begin{array}{lll}
30 & & \\
& 20 & \\
& & 0
\end{array}\right] .
\end{aligned}
$$

Expression of vector $V$ obtained by (9) can be given as follows:

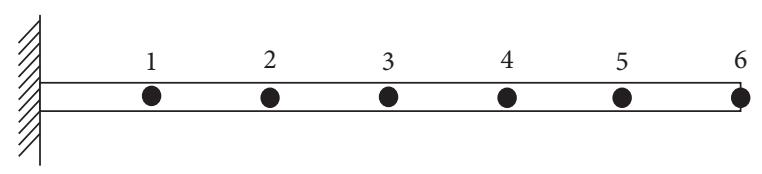

FIgURE 2: Cantilever beam modal test model.

TABle 1: Cantilever beam physical parameters.

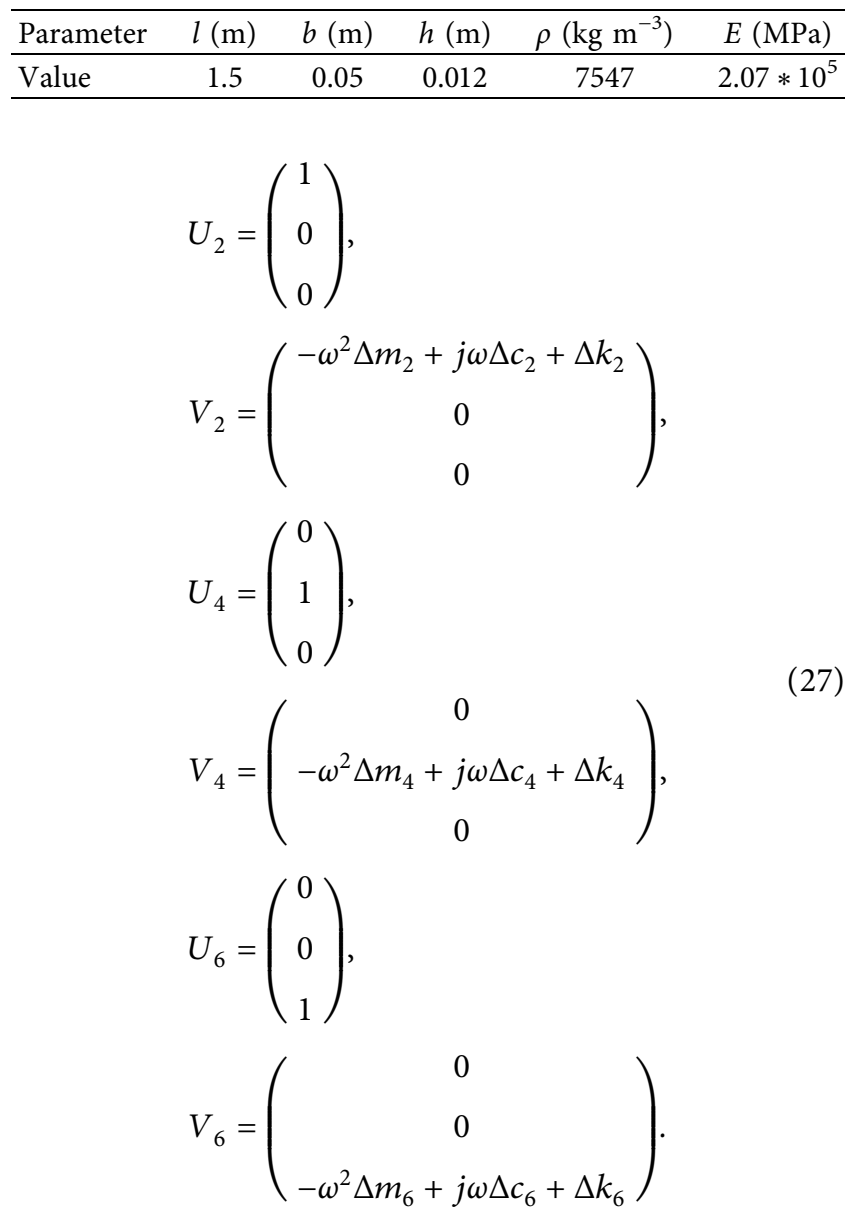

According to (13), receptances $H$ of original structure can be obtained.

$$
H=-\frac{H a}{\omega^{2}}=\left[\begin{array}{lll}
H_{22} & H_{24} & H_{26} \\
H_{42} & H_{44} & H_{46} \\
H_{62} & H_{64} & H_{66}
\end{array}\right] .
$$

The accelerances $H^{*}$ of modified structure can be calculated by (11) and (14).

$$
\begin{aligned}
H^{*} & =H-H\left[\begin{array}{lll}
U_{2} & U_{4} & U_{6}
\end{array}\right] W^{-1}\left[\begin{array}{lll}
V_{2} & V_{4} & V_{6}
\end{array}\right]^{T} H, \\
W & =\left[\begin{array}{ccc}
1+V_{2}^{T} H U_{2} & V_{2}^{T} H U_{4} & V_{2}^{T} H U_{6} \\
V_{4}^{T} H U_{2} & 1+V_{4}^{T} H U_{4} & V_{4}^{T} H U_{6} \\
V_{6}^{T} H U_{2} & V_{6}^{T} H U_{4} & 1+V_{6}^{T} H U_{6}
\end{array}\right] .
\end{aligned}
$$

It can be seen from the above that all the FRFs $H$ (corresponding to original structure) required for 




(a)

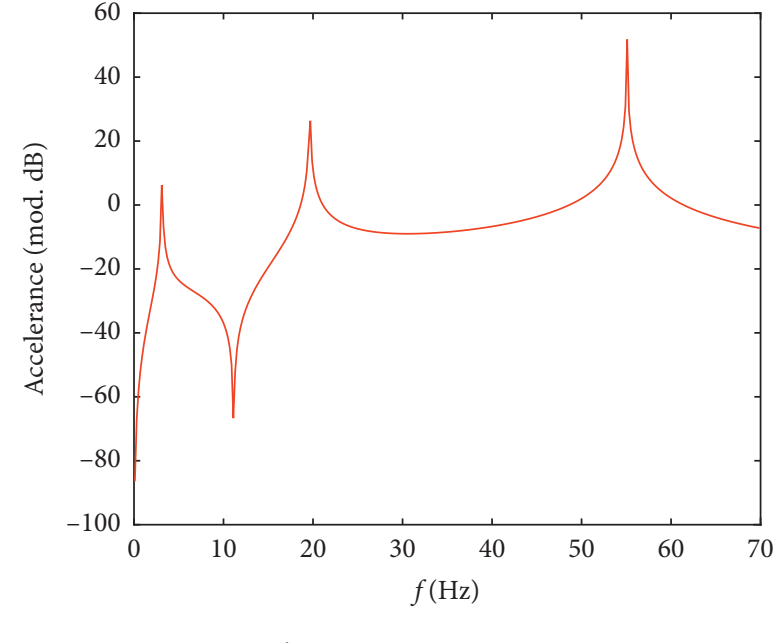

(b)

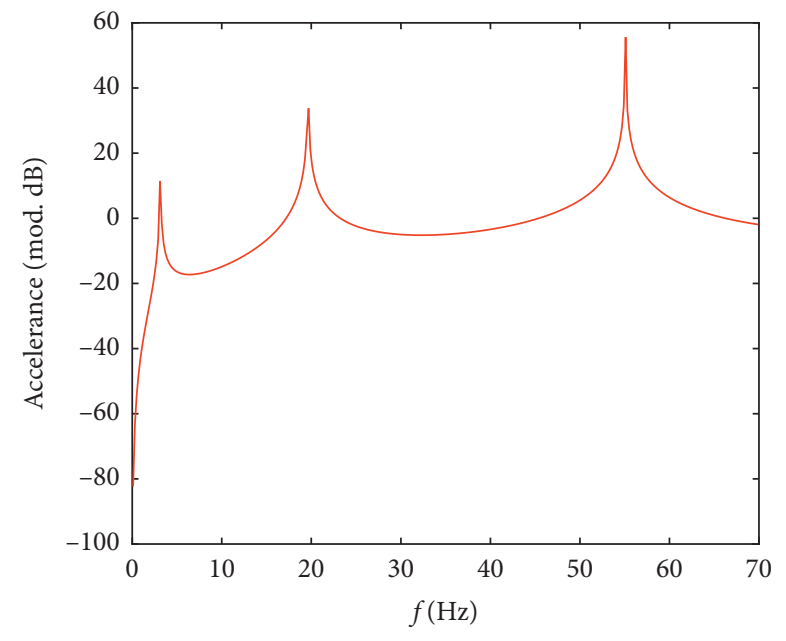

— Ha-ori-lp

(c)

FIGURE 3: Accelerances of original structural, (a) original accelerance $H a_{22}$, (b) original accelerance $H a_{24}$, and (c) original accelerance $H a_{26}$.

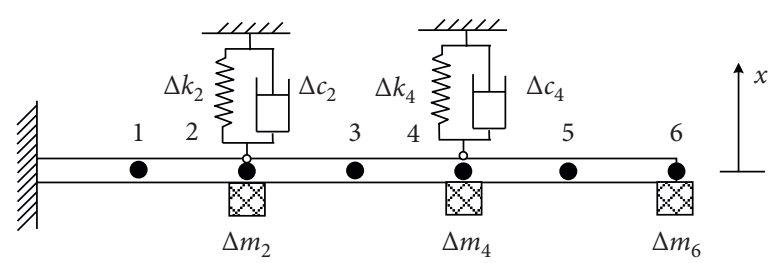

Figure 4: Adding mass, stiffness, and damping.

calculating $H^{*}$ (corresponding to modified structure) are related to the modification coordinates. Then, accelerances $H a^{*}$ of modified structure can be directly calculated by (13).

$$
H a^{*}=-\omega^{2} H^{*}=\left[\begin{array}{lll}
H a_{22}^{*} & H a_{24}^{*} & H a_{26}^{*} \\
H a_{42}^{*} & H a_{44}^{*} & H a_{46}^{*} \\
H a_{62}^{*} & H a_{64}^{*} & H a_{66}^{*}
\end{array}\right] .
$$

Although nine FRFs are calculated, only three of them are shown for the sake of brevity, as can be seen in Figure 5 .

As shown in Figure 5, "exact" represents the exact accelerances curves obtained directly by numerical calculation. "Measured" represents the accelerances curves obtained by the method this paper proposed. "Original" represents the accelerances curves of original structure. 


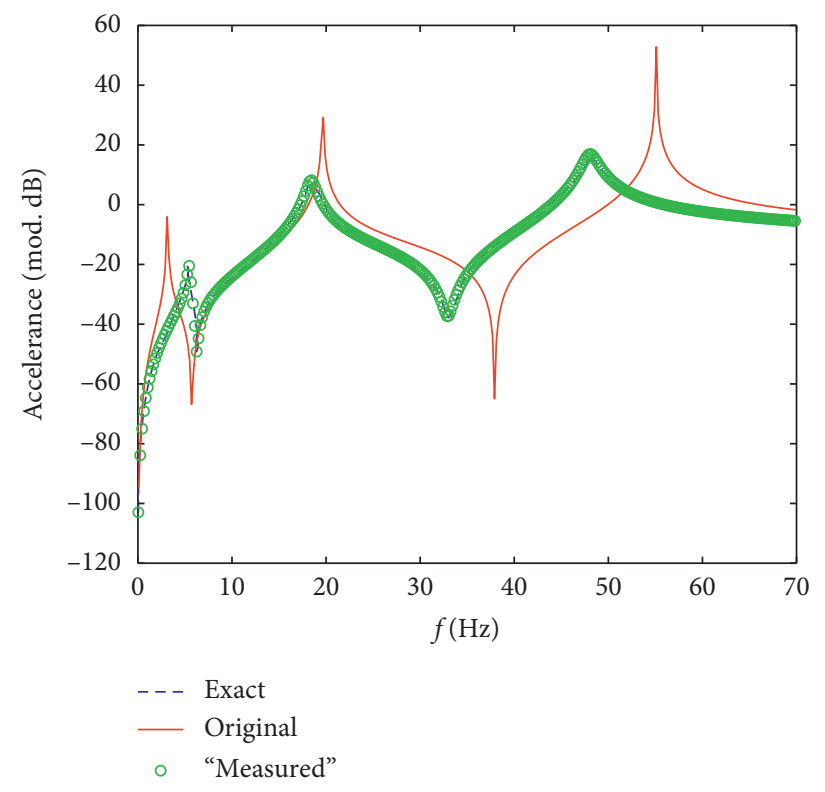

(a)

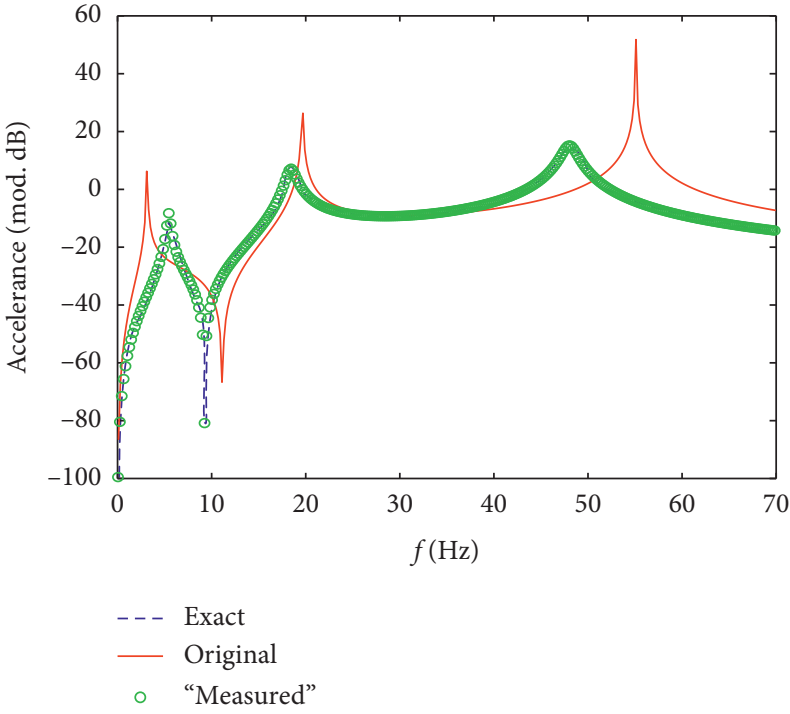

(b)

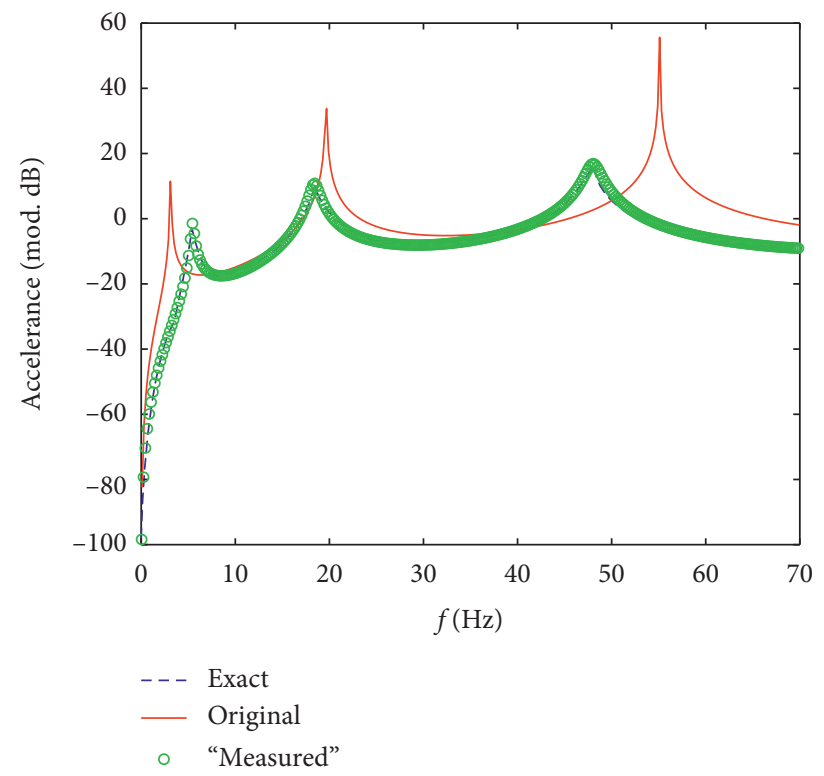

(c)

Figure 5: Comparison of exact, original, and "measured" accelerances, (a) accelerances $H a_{22}^{\prime}, H a_{22}$ and $H a_{22}^{*}$, (b) accelerances $H a_{24}^{\prime}, H a_{24}$ and $H a_{24}^{*}$, and (c) accelerances $H a_{26}^{\prime}, H a_{26}$ and $H a_{26}^{*}$.

It is obvious from Figure 5 that, after dynamic modification to the original structure of vibration system, the accelerances curves "Measured" as a whole are in quite good agreement with those of target accelerances curve "exact", which indicates that the results of these two methods are consistent and accuracy of the method is verified.

3.3. Adding Spring-Mass Substructure to the Original Structure. One and multiple spring-mass substructures are, respectively, added to the original structure to calculate the FRFs of the modified structure for verification of the proposed method.
3.3.1. Adding One Spring-Mass Substructure to the Original Structure. As shown in Figure 6, a spring-mass substructure is added to the original structure at coordinate 1 , where the stiffness $\Delta k_{1}$ of the substructure is $6000 \mathrm{~N} / \mathrm{m}$, and the mass $\Delta m_{1}$ of the substructure is $0.5 \mathrm{~kg}$. For the accelerances of the structure after adding this spring-mass substructure, 4 natural frequencies are obtained within the range of $0-70 \mathrm{~Hz}$. Response points are chosen at coordinates 1, 3, and 5. Hammer impact is moving sequentially from points 1,3 to 5 for "measuring" accelerances $H a=\left[\begin{array}{lll}H a_{11} & H a_{13} & H a_{15} \\ H a_{31} & H a_{33} & H a_{35} \\ H a_{51} & H a_{35} & H a_{55}\end{array}\right]$. The goal of this example is to calculate the nine accelerances 


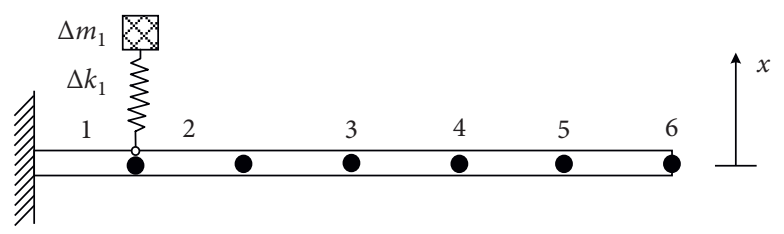

FIGURE 6: Adding one spring-mass substructure to the original structure.

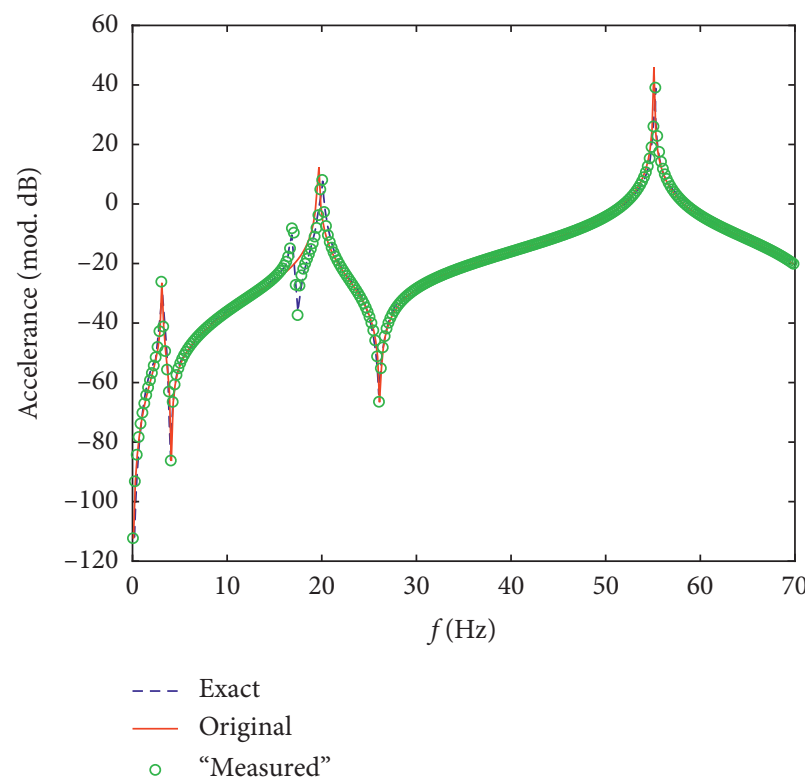

(a)

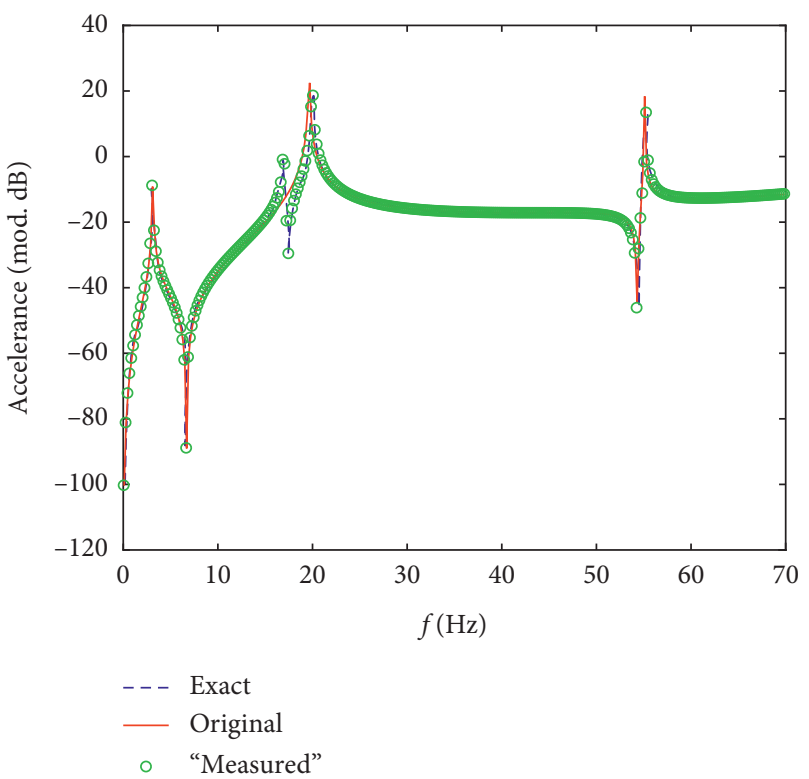

(b)

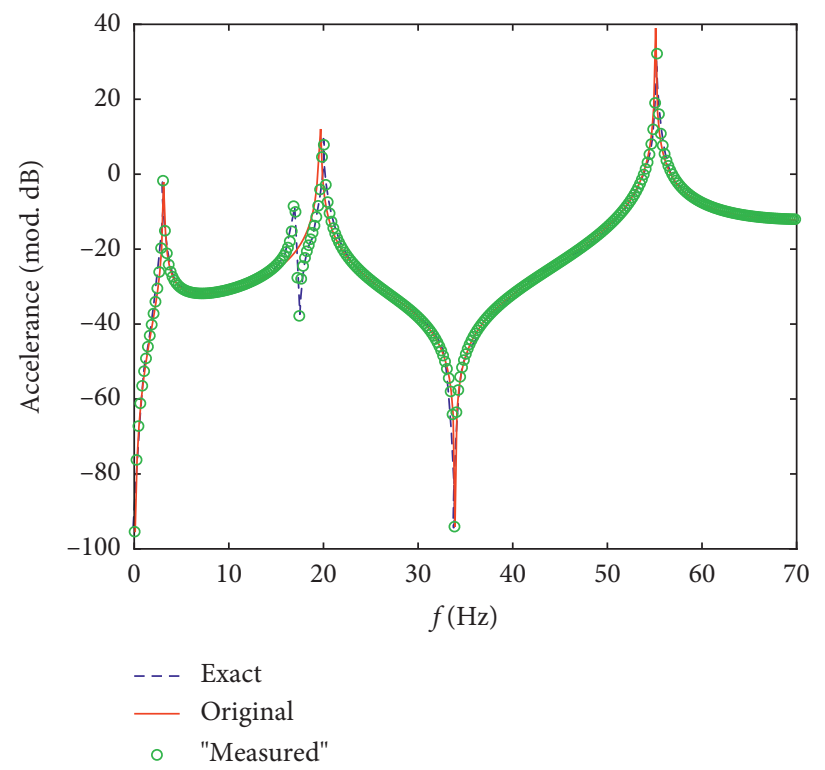

(c)

Figure 7: Comparison of exact, original, and "measured" accelerances, (a) accelerances $H a_{11}^{\prime}, H a_{11}$ and $H a_{11}^{*}$, (b) accelerances $H a_{13}^{\prime}, H a_{13}$ and $H a_{13}^{*}$, and (c) accelerances $H a_{15}^{\prime}, H a_{15}$ and $H a_{15}^{*}$.

$H a^{*}=\left[\begin{array}{lll}H a_{11}^{*} & H a_{13}^{*} & H a_{15}^{*} \\ H a_{31}^{*} & H a_{33}^{*} & H a_{35}^{*} \\ H a_{51}^{*} & H a_{35}^{*} & H a_{55}^{*}\end{array}\right]$. Similarly, only three of them are shown for the sake of simplicity, as can be seen in Figure 7.
As shown in Figure 7, as one spring-mass substructure is added to the original structure, the exactly calculated accelerances curves of the modified structure and the ones calculated by the proposed method completely coincide. 


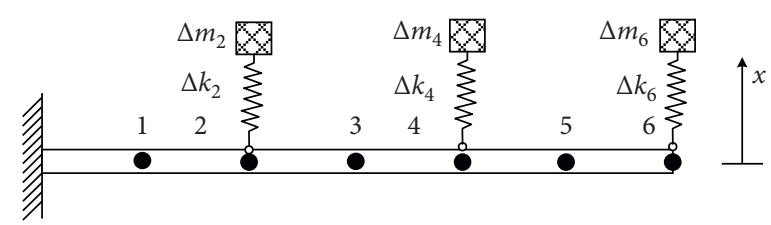

FIgURE 8: Adding three spring-mass substructures to the original structure.

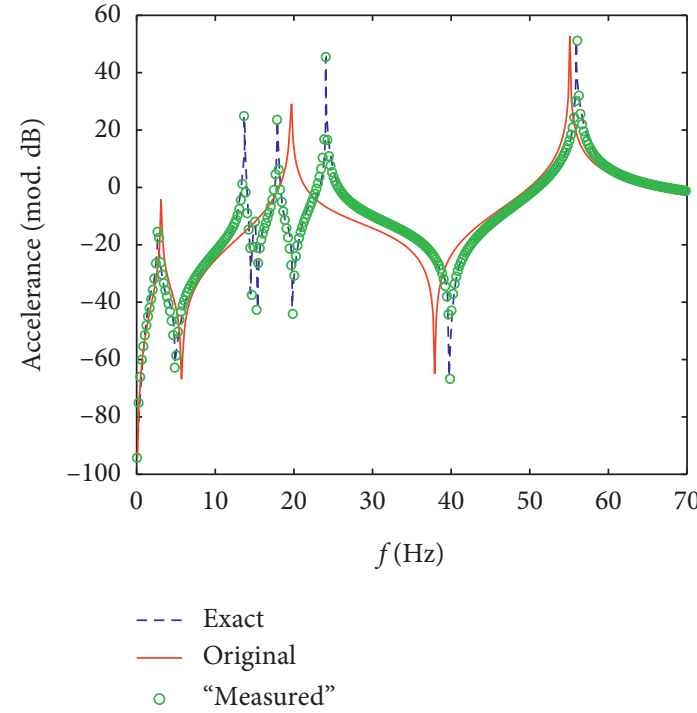

(a)

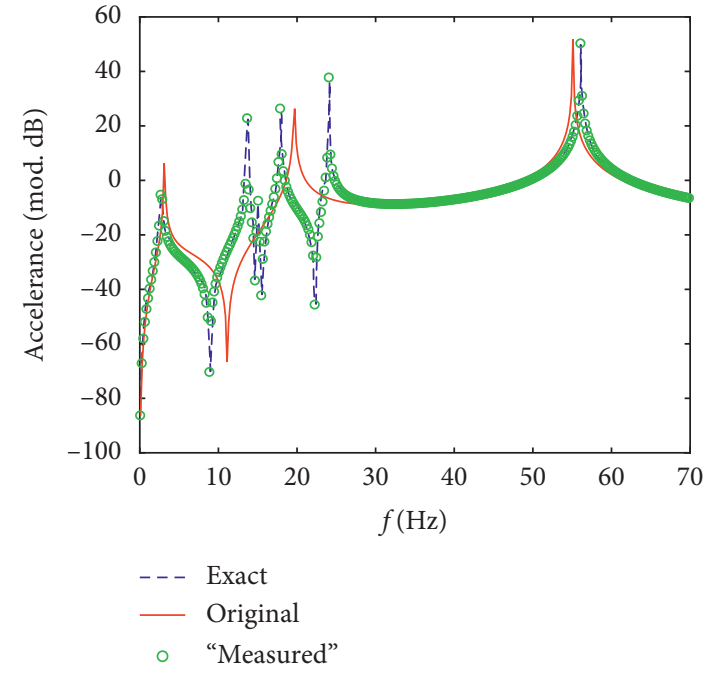

(b)



(c)

Figure 9: Comparison of exact, original, and "measured" accelerances, (a) accelerances $H a_{22}^{\prime}, H a_{22}$ and $H a_{22}^{*}$, (b) accelerances $\mathrm{Ha}_{24}^{\prime}, \mathrm{Ha}_{24}$ and $\mathrm{Ha}_{24}^{*}$, and (c) accelerances $\mathrm{Ha}_{26}^{\prime}, \mathrm{Ha}_{26}$ and $\mathrm{Ha}_{26}^{*}$.

This result indicates that, in the condition of adding one spring-mass substructure, modified accelerances can be accurately calculated by the proposed method.

3.3.2. Adding Three Spring-Mass Substructures to the Original Structure. Figure 8 is a cantilever beam model after adding substructures. Three spring-mass substructures are added at coordinate points 2,4 , and 6 , respectively. The masses $\Delta m_{2}, \Delta m_{4}$, and $\Delta m_{6}$ of these substructures are $0.35 \mathrm{~kg}, 0.42 \mathrm{~kg}$, and $0.39 \mathrm{~kg}$, respectively, and stiffness $\Delta k_{2}$, $\Delta k_{4}$ and $\Delta k_{6}$ of these substructures are $3000 \mathrm{~N} / \mathrm{m}, 4000 \mathrm{~N} / \mathrm{m}$, and $5000 \mathrm{~N} / \mathrm{m}$, respectively.

According to (24) and (25), the vector $U$ and $V$ can be written as follows. 


$$
\begin{aligned}
& U_{2}=\left(\begin{array}{l}
1 \\
0 \\
0
\end{array}\right) \text {, } \\
& V_{2}=\left(\begin{array}{c}
-\frac{\omega^{2} \Delta m_{2} \Delta k_{2}}{\Delta k_{2}-\omega^{2} \Delta m_{2}} \\
0 \\
0
\end{array}\right) \text {, } \\
& U_{4}=\left(\begin{array}{l}
0 \\
1 \\
0
\end{array}\right) \text {, } \\
& V_{4}=\left(\begin{array}{c}
0 \\
-\frac{\omega^{2} \Delta m_{4} \Delta k_{4}}{\Delta k_{4}-\omega^{2} \Delta m_{4}} \\
0
\end{array}\right), \\
& U_{6}=\left(\begin{array}{l}
0 \\
0 \\
1
\end{array}\right) \text {, } \\
& V_{6}=\left(\begin{array}{c}
0 \\
0 \\
-\frac{\omega^{2} \Delta m_{6} \Delta k_{6}}{\Delta k_{6}-\omega^{2} \Delta m_{6}}
\end{array}\right)
\end{aligned}
$$

Response points are chosen at coordinates 2, 4, and 6 . Hammer impact is moving sequentially from points 2,4 to 6 .

Then accelerances $H a=\left[\begin{array}{lll}H a_{22} & H a_{24} & H a_{26} \\ H a_{42} & H a_{44} & H a_{46} \\ H a_{62} & H a_{64} & H a_{66}\end{array}\right]$ can be

"measured". The accelerances to be calculated are $H a^{*}=\left[\begin{array}{lll}H a_{22}^{*} & H a_{24}^{*} & H a_{26}^{*} \\ H a_{42}^{*} & H a_{44}^{*} & H a_{46}^{*} \\ H a_{62}^{*} & H a_{64}^{*} & H a_{66}^{*}\end{array}\right]$. Only the curves of $H a_{22}^{*}, H a_{22}^{*}$ and $H a_{22}^{*}$ are shown for the sake of brevity, as can be seen in Figure 9.

As shown in Figure 9, after adding three spring-mass substructures to the original structure, accelerances curves obtained by the proposed method coincide with the exactly values, which proves the accuracy of this method.

\section{Conclusion}

The work presented in this paper dealt with the problem of reanalyzing FRFs of a modified structure. A method is developed by employing SMW formula based on the FRFs related to the modification coordinates of original system. This method can solve the calculation problem of FRFs of modified structure in only one step. The accuracy and efficiency of this method are proved according to the simulated modal testing of cantilever beam model.

Two kinds of examples are used in verification. One is directly adding additional masses, stiffnesses, and damping to the cantilever beam. This kind of modification is mainly for the case that internal structural parameters matrices are changed. The other is adding spring-mass substructures to the cantilever beam. This condition is suitable for models whose original structure is immutable. Both numerical examples demonstrated good performance in the simulation verification. The results show that this approach is suitable for multiple-element change situation of structural modification and could improve the efficiency of structural reanalysis.

\section{Data Availability}

The data used to support the findings of this study are available from the corresponding author upon request.

\section{Conflicts of Interest}

The authors declare that they have no conflicts of interest.

\section{Acknowledgments}

This work was supported by the Hubei Provincial Natural Science Foundation of China (2017CFB128), the Doctoral Scientific Research Foundation of Hubei University of Technology (BSQD14027), and Open fund of Hubei Key Laboratory of Modern Manufacturing Quality Engineering (KFJJ-2020003).

\section{References}

[1] S.-H. Tsai, H. Ouyang, and J.-Y. Chang, "Inverse structural modifications of a geared rotor-bearing system for frequency assignment using measured receptances," Mechanical Systems and Signal Processing, vol. 110, pp. 59-72, 2018.

[2] D. J. Ewins, Modal Testing: Theory, Practice and Applications, Research Studies Press, England, UK, 2nd edition, 2000.

[3] J. He and Y. Li, "Relocation of anti-resonances of a vibratory system by local structural changes," International Journal of Analytical and Experimental Modal Analysis, vol. 10, no. 3, pp. 224-235, 1995.

[4] K. T. Joseph, "Inverse eigenvalue problem in structural design," AIAA Journal, vol. 30, no. 12, pp. 2890-2896, 1992.

[5] Y. M. Ram and S. G. Braun, "An inverse problem associated with modification of incomplete dynamic systems," Journal of Applied Mechanics, vol. 58, no. 1, pp. 223-237, 1991.

[6] Y. G. Tsuei and E. K. L. Yee, "A method for modifying dynamic properties of undamped mechanical systems," Journal 
of Dynamic Systems, Measurement, and Control, vol. 111, no. 3, pp. 403-408, 1989.

[7] J. F. Baldwin and S. G. Hutton, "Natural modes of modified structures," AIAA Journal, vol. 23, no. 11, pp. 1937-1743, 1985.

[8] J.-F. M. Barthelemy and R. T. Haftka, "Approximation concepts for optimum structural design-a review," Structural Optimization, vol. 5, no. 3, pp. 129-144, 1993.

[9] B. P. Wang and W. D. Pilkey, "Eigenvalue reanalysis of locally modified structures using a generalized Rayleigh's method," AIAA Journal, vol. 24, no. 6, pp. 983-990, 1986.

[10] W. M. To and D. J. Ewins, "Structural modification analysis using Rayleigh quotient iteration," International Journal of Mechanical Sciences, vol. 32, no. 3, pp. 169-179, 1990.

[11] S. H. Chen, X. W. Yang, and H. D. Lian, "Comparison of several eigenvalue reanalysis methods for modified structures," Structural and Multidisciplinary Optimization, vol. 20, no. 4, pp. 253-259, 2000.

[12] Z. Li, B. S. Wu, and H. F. Liu, "Reanalysis method after structural boundary constraint modification," Journal of Computational Mechanics, vol. 29, no. 5, pp. 699-703, 2012.

[13] H. F. Liu, B. S. Wu, C. W. Lim, and Z. G. Li, "An approach for structural static reanalysis with unchanged number of degrees of freedom," Structural and Multidisciplinary Optimization, vol. 45, no. 5, pp. 681-692, 2012.

[14] Q. Song, P. Chen, and S. Sun, "An exact reanalysis algorithm for local non-topological high-rank structural modifications in finite element analysis," Computers \& Structures, vol. 143, pp. 60-72, 2014.

[15] G. Huang, H. Wang, and G. Li, "A reanalysis method for local modification and the application in large-scale problems," Structural and Multidisciplinary Optimization, vol. 49, no. 6, pp. 915-930, 2014.

[16] H. F. Liu, B. S. Wu, and Z. G. Li, "Method of updating the Cholesky factorization for structural reanalysis with added degrees of freedom," Journal of Engineering Mechanics, vol. 140, no. 2, pp. 384-392, 2014.

[17] D. Materna and V. K. Kalpakides, "Nonlinear reanalysis for structural modifications based on residual increment approximations," Computational Mechanics, vol. 57, no. 1, pp. 1-18, 2016.

[18] Y.-S. Kim and H.-C. Eun, "Seismic performance evaluation of footing-to-circular RC pier connection reinforced by highmanganese steel bars (HMSBs)," Advances in Civil Engineering, vol. 2018, Article ID 3084078, 9 pages, 2018.

[19] S. P. Zheng, B. S. Wu, and Z. G. Li, "Free vibration reanalysis of structures with added degrees of freedom," Computers \& Structures, vol. 206, pp. 31-41, 2018.

[20] U. Krisch, "Combined approximations-a general reanalysis approach for structural optimization," Structural and Multidisciplinary Optimization, vol. 20, no. 2, pp. 97-106, 2000.

[21] U. Kirsch, M. Bogomolni, and I. Sheinman, "Nonlinear dynamic reanalysis of structures by combined approximations," Computer Methods in Applied Mechanics and Engineering, vol. 195, no. 33-36, pp. 4420-4432, 2006.

[22] W. Zuo, T. Xu, H. Zhang, and T. Xu, "Fast structural optimization with frequency constraints by genetic algorithm using adaptive eigenvalue reanalysis methods," Structural and Multidisciplinary Optimization, vol. 43, no. 6, pp. 799-810, 2011.

[23] R. Sun, D. Liu, T. Xu, H. Zhang, and W. Zuo, "New adaptive technique of Kirsch method for structural reanalysis," AIAA Journal, vol. 52, no. 3, pp. 486-495, 2014.
[24] W. Zuo, J. Bai, and J. Yu, "Sensitivity reanalysis of static displacement using Taylor series expansion and combined approximate method," Structural and Multidisciplinary Optimization, vol. 53, no. 5, pp. 953-959, 2016.

[25] W. Zuo, K. Huang, J. Bai, and G. Guo, "Sensitivity reanalysis of vibration problem using combined approximations method," Structural and Multidisciplinary Optimization, vol. 55, no. 4, pp. 1399-1405, 2017.

[26] J. Sherman and W. J. Morrison, "Adjustment of an inverse matrix corresponding to a change in one element of a given matrix," The Annals of Mathematical Statistics, vol. 21, no. 1, pp. 124-127, 1950.

[27] M. A. Woodbury, Inverting Modified Matrices, Statistical Research Group, Princeton University, Princeton, NJ, USA, 1950.

[28] M. A. Akgün, J. H. Garcelon, and R. T. Haftka, "Fast exact linear and non-linear structural reanalysis and the ShermanMorrison-Woodbury formulas," International Journal for Numerical Methods in Engineering, vol. 50, no. 7, pp. 15871606, 2001.

[29] Z. Liu, W. Li, H. Ouyang, and D. Wang, "Eigen structure assignment in vibrating systems based on receptances," Archive of Applied Mechanics, vol. 85, no. 6, pp. 713-724, 2015. 